\title{
Variables deportivas y personales en la ocurrencia de lesiones deportivas. Diferencias entre deportes individuales y colectivos Sport and personal variables in the occurrence of sports injuries. Differences between individual and team sports \\ Joel Manuel Prieto Andreu \\ Universidad CEU Cardenal Herrera de Castellón (España)
}

Resumen. El propósito del presente estudio fue determinar la relación entre factores deportivos y personales con la incidencia de lesión (frecuencia y gravedad) en deportes individuales (natación, atletismo y tenis), y en deportes colectivos (fútbol, balonmano y baloncesto). La muestra total del estudio fue de 453 sujetos (47 no lesionados), 285 hombres (23 no lesionados) y 168 mujeres (24 no lesionadas) de diferentes Clubes Deportivos de la Comunidad Valenciana. El estudio se basa en una metodología cuantitativa, y su diseño fue descriptivo, observacional y correlacional. Para evaluar las lesiones, se elaboró un registro de auto-informe. Los resultados indicaron que un mayor número de lesiones totales y un mayor índice de lesión se asociaron con una menor edad $(\mathrm{p}<.05)$, con una mayor experiencia $(\mathrm{p}<.01)$ y con deportes colectivos $(\mathrm{p}<.01)$, no encontrándose relación con el género. Se señala la aplicabilidad de los resultados y se señalan conclusiones del estudio.

Palabras clave. Lesiones, factores deportivos, factores personales, lesión, deportes individuales, deportes colectivos.

Abstract. The purpose of the study was to analyze the association between the sport and personal factors and the incidence of injuries (frequency and severity) according to individual (swimming, tennis and athletics) and team sports (basketball, handball and football). The sample comprised 453 athletes (47 non-injured), 285 males (23 non-injured) and 168 females (24 non-injured) recruited from sports clubs in Valencia. The study design was quantitative, descriptive, cross-sectional and correlational. Injuries were evaluated by self-report. The results indicated that a greater number of total injuries and a higher injury rates were associated with athletes of a younger age $(\mathrm{p}<.05)$, more experienced athletes ( $<<.01)$ and team-sport athletes $(\mathrm{p}<.01)$. Gender was unrelated to injuries. Practical applications of the results are mentioned and some conclusions of the study are indicated. Keywords. Sport injuries, sport factors, personal factors, injury, individual sports, team sports.

\section{Introducción}

Considerar los factores que pueden propiciar que un deportista se lesione ha adquirido una gran importancia debido, fundamentalmente, al aumento de las exigencias deportivas en los deportistas de alto rendimiento (Bahr y Krosshaug, 2005; Ekstrand, Walden, y Häglund, 2009). La evidencia empírica indica que factores psicológicos, como el estrés, parecen desempeñar un importante papel en la ocurrencia de lesiones y en la recuperación de la lesión (Buceta, 2008; Martínez-Romero, 2008; Naylor, 2008; Palmeira, 1998; Spano, 2008). Por otra parte, factores físicos como el exceso de entrenamiento y la fatiga física, son las causas principales de lesiones en la práctica deportiva competitiva (Bahr y Krosshaug, 2005).

Bahr y Krosshaug (2005) indican que para explicar todos los factores de riesgo internos y externos, así como el mecanismo de aparición de la lesión, es necesario un acercamiento multidimensional. Van Mechelen y Kemper (1992), en su modelo sobre la causalidad de la lesión(Figura 1), indican que aunque la lesión puede haberse producido por un solo

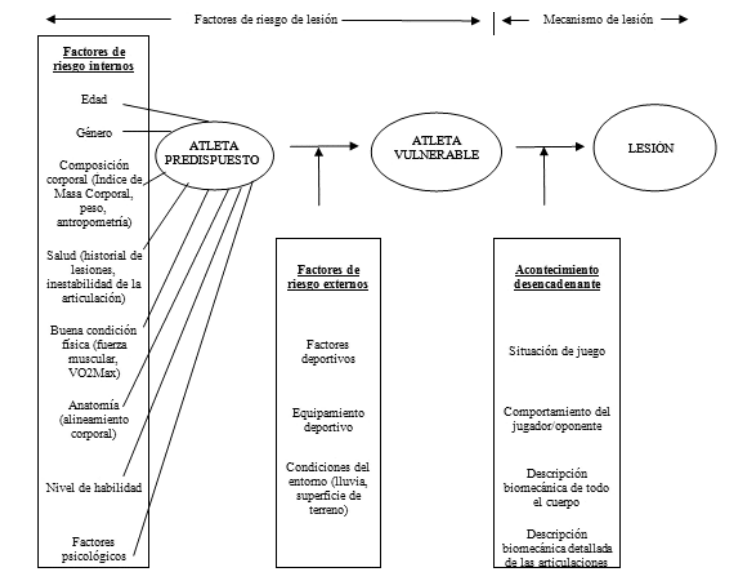

Figura 1. "Modelo comprensivo sobre la causalidad de la lesión". Tomado de Van Mechelen y Kemper (1992)

Fecha recepción: 08-10-14- Fecha envío revisores: 26-10-15- Fecha de aceptación: 10-03-15 Joel Prieto Andreu

Joel_manuel.prieto@uch.ceu.es acontecimiento, puede resultar de la interacción compleja entre factores de riesgo internos (edad, género, etc.) y externos (modalidad deportiva, experiencia, horas de entrenamiento, etc.). En esta línea se manifiestan los estudios realizados por diferentes investigadores (Hanson et al., 1992; Heil, 1993; Palmi, 2001), coincidiendo en agrupar los factores causantes de las lesiones deportivas en factores internos y externos. En general, se puede considerar que la etiología de la lesión es multifactorial, con factores de tipo deportivo, personal, físico, deportivo y psicológiCo.

Resulta importante conocer los factores determinantes que afectan a la lesión deportiva para poder realizar una labor eficaz de prevención. No obstante, hay otros factores que también pueden intervenir, la conducta de los oponentes y compañeros, factores deportivos: la modalidad deportiva y la experiencia deportiva, factores físicos como el género, o el exceso de entrenamiento y la fatiga física, consideradas como las principales causas de lesiones relacionadas con el deporte y el ejercicio (Hanson et al., 1992). Por otro lado, en el estudio de Rodal, García y Arufe (2013) encontraron que variables como el volumen de entrenamiento y las lesiones previas se correlacionan positivamente con la variable dependiente «lesiones en 12 semanas». Sin embargo, en un estudio que relacionaba lesiones, género y edad de diferentes deportistas del Oeste de Australia, se indica que eran incapaces de determinar en quémedida las lesiones eran debidas al sobreentrenamiento(Stevenson, Hamer y Finch, 2000).

Respecto a la relación entre modalidad deportiva y lesiones deportivas, dentro de los sistemas de entrenamiento de unas especialidades y otras, es posible que en algunos deportes se incremente el riesgo de lesión por que se necesite entrenar varias horas al día y durante todo el año, mientras que en otros deportes una dedicación baja puede dar buenos rendimientos, siendo bajos los niveles de exigencia. Sin embargo Jan, Margareta, Birgitta y Sten-Otto (1983) aunque señalan que la competición a nivel de elite produce lesiones más graves, sugieren que el diseño del entrenamiento está estrechamente relacionado con el número de lesiones y que los equipos con más promedio de entrenamiento tienen pocas lesiones y es el diseño más acertado. Los resultados de Jan et al. (1983) muestran que equipos con menos media de entrenamiento (1400 horas de entrenamiento) tenían más lesiones que los equipos que entrenaban más $(\mathrm{p}<.05)$.

Como afirman Bahr y Krosshaug (2005), los factores intrínsecos suelen actuar de forma simultánea con los extrínsecos en el momento 
que ocurre la lesión y, actuando de forma aislada, raras veces llegan a ser suficientes para provocar lesión. Sin embargo, la combinación de ambos tipos de factores de riesgo y su interacción predisponen al deportista a lesionarse. Por esta razón, se piensa que desde una perspectiva multifactorial, atendiendo a factores internos y externos, los resultados de este estudio se podrían utilizar para identificar aquellos factores que posibilitan una mayor vulnerabilidad del deportista a lesionarse; y como consecuencia a lo anterior, para poder diseñar programas de intervención que disminuyeran los riesgos de sufrir lesión.

En este estudio se planteó como objetivo, determinar la relación entre el número de lesiones totales (frecuencia y gravedad) con las variables sociodemográficas (edad, género) y con las variables deportivas (experiencia, hora de entrenamiento y modalidad deportiva).

\section{Método}

\section{Diseño}

El diseño utilizado en la investigación fue de corte transversalretrospectivo, descriptivo y correlacional (Thomas y Nelson, 2007). Las variables dependientes fueron: a) número de lesiones según gravedad, y b) índice de lesión (número total de lesiones dividido entre el número de horas de entrenamiento semanal). Las variables independientes fueron: a) edad, b) género, c) años de experiencia, y d) modalidad deportiva (colectivo o individual). En este estudio se ha considerado distribuir la muestra según el género (masculino y femenino) y según el tipo de deporte, colectivo (balonmano, baloncesto y fútbol) e individual (atletismo, natación, tenis).

En la tabla 1 se puede observar la media de edad y el recuento de la experiencia deportiva y del tiempo dedicado en horas por semana de los deportistas, diferenciándolos entre deportistas de deportes individuales y colectivos.

\section{Participantes}

La muestra total del estudio fue de 452 sujetos (46 no lesionados), 284 hombres (39 no lesionados) y 168 mujeres (7 no lesionadas). Los hombres tenían una media de edad de 21,77 años (DT=4.81) y las mujeres una media de edad de 20,55 años (DT=4.39). Los criterios de inclusión utilizados para seleccionar la muestra fueron los siguientes: varones y mujeres federados que estuvieran compitiendo en al actualidad. La muestra estuvo formada por deportistas individuales (deportistas federados de atletismo (61), natación (121) y tenis (72)), 143 hombres (125 lesionados) y 111 mujeres (90 lesionadas), y por deportistas colectivos (deportistas federados de fútbol (79), baloncesto (55) y balonmano (64)), 141 hombres (137 lesionados) y 57 mujeres (54 lesionadas). El número de deportistas lesionados graves o muy grave fue de 209; el número de deportistas con lesiones moderadas fue de 275; el número de deportistas con lesiones leves fue de 300; y el número de deportistas no lesionados fue de 46.

\section{Instrumentos}

Para la evaluación de las lesiones deportivas (historial de lesiones, gravedad y frecuencia de lesión) se utilizó un Cuestionario de autoinforme realizado ad hoc para el estudio (ver Anexo I) a partir del utilizado por Díaz (2002). En este estudio, se agruparon las lesiones acontecidas en las dos últimas temporadas según su gravedad, siguiendo el modelo de agrupación de lesión de Heil (1993): lesiones leves (requieren atención o tratamiento pero no se interrumpe la actividad deportiva); lesiones moderadas (es necesario el tratamiento y limitan la participación deportiva); lesiones graves (implican una interrupción prolongada de la actividad, a menudo con hospitalizaciones e intervenciones quirúrgicas); lesiones graves que provocan un deterioro crónico (imprescindible la rehabilitación permanente); y lesiones graves que provocan una incapacidad permanente (suponen el abandono deportivo).

Respecto a la frecuencia de lesión, en este estudio se utilizaron dos variables relacionadas con el número de lesiones: número total de lesiones (la suma del número de lesiones leves, moderadas, graves y muy graves); e índice de lesión (número total de lesiones dividido entre el número de horas de entrenamiento semanal).

Por último, se elaboró un cuestionario de recogida de información, incorporando las sugerencias de otros autores (Buceta, 1996; Olmedilla, Ortega, y Abenza, 2007; Van Mechelen et al., 1996), dónde se contemplaron aspectos sociodemográficos y personales cómo el género, peso, estatura, deporte practicado, posesión de licencia federativa, club de pertenencia, tiempo de entrenamiento y experiencia (ver Anexo II).

\section{Procedimiento}

Se acudió a los entrenamientos durante el periodo 2011-2012, y antes de iniciar el proceso de administración de los cuestionarios se solicitó permiso a los propios deportistas, informándoles de la confidencialidad y del anonimato de los datos, y solicitándoles la firma del documento de consentimiento informado. Se administraron los cuestionarios de forma individual o colectiva en los mismos Clubes de la Comunidad Valenciana.

\section{Análisis estadístico}

Se utilizó el paquete estadístico SPSS 15.0. Se realizaron análisis descriptivos por un lado, y por otro lado, análisis de regresión lineal para determinar la influencia de las variables psicológicas y sociodemográficas en la frecuencia y la gravedad de lesión, obteniéndose una varianza que ayudó a predecir de forma significativa esa influencia. Todos los análisis estadísticos se realizaron con un nivel de significación de pd».05.

\section{Resultados}

En primer lugar, se realizó un análisis descriptivo, en la tabla 2 se puede observar el recuento de las variables, diferenciando entre deportes individuales y colectivos.

En la tabla 3 se aprecia una regresión lineal, donde se relacionan los factores deportivos y personales con el número total de lesiones. Los datos de la tabla 3 señalan que las variables que más contribuyen en la varianza (30.90\%) son, edad, tipo de modalidad y la experiencia. La combinación de estas variables predice de forma significativa el índice de lesiones $\left(\mathrm{F}_{(8.443)}=26.23, \mathrm{p}<.001\right)$.

Tabla 1

Media de edad y recuento del tiempo dedicado yexperiencia deportiv

\begin{tabular}{cccc} 
Media de edad y recuento del tiempo dedicado yexperiencia deportiva \\
\hline & & Deporte Individual & Deporte Colectivo \\
\hline & Edad & 2.17 & 22.79 \\
\hline & 4 año & 7 & 0 \\
Tiempo total jugando & $1-4$ añ os & 63 & 0 \\
(experiencia deportiva) & $4-8$ añ os & 126 & 51 \\
& $8-12$ años & 40 & 63 \\
& $>12$ años & 18 & 84 \\
\hline \multirow{4}{*}{ Tiempo dedicado en } & $<$ hora & 14 & 0 \\
horas por semana & $1-3$ horas & 40 & 43 \\
& $3-5$ horas & 31 & 27 \\
& $5-8$ horas & 56 & 62 \\
& $>8$ horas & 113 & 66 \\
\hline
\end{tabular}

Tabla 2
Medias de las variables según moda lidad deportiva

Edad
Índice de lesión

Indice de lesión
Número total de lesiones Número de lesiones leves Número delesiones moder Número de lesiones graves Número de lesiones muy graves DT: Desviación Típica

Un 38.2\% de la varianza puede ser predicha por estos factores deportivos y personales. La combinación de estas variables predice de forma significativa el número total de lesiones $\left(\mathrm{F}_{(8.433)}=.25, \mathrm{p}<.001\right)$. En la tabla 3 se puede observar como a menor edad mayor número de lesiones totales $(\beta=-18, \mathrm{p}<.001)$. De igual forma, se aprecia que a mayor experiencia deportiva mayor número de lesiones totales $(\beta=-$ $.27, \mathrm{p}<.001)$. Por otro lado, existen diferencias significativas con respecto al tipo de modalidad deportiva (deporte individual o deporte colectivo) siendo los deportistas de equipo los que tuvieron mayor número de lesiones totales (5.68/2.35) $(\beta=-.28, p<.001)$. El género no tuvo relación significativa con el número total de lesiones. 
En la tabla 4 se observa una regresión lineal, donde se relacionan los factores deportivos y personales con el índice de lesión. Los datos de la tabla 4 señalan que las variables que más contribuyen en la varianza (32.4\%) son, edad, la experiencia y tipo modalidad. La combinación de estas variables predice de forma significativa el índice de lesiones $\left(\mathrm{F}_{(8.443)}=26.23, \mathrm{p}<.001\right)$.

Un $43.60 \%$ de la varianza puede ser predicha por estos factores deportivos y personales. La combinación de estas variables predice de forma significativa el número total de lesiones $\left(\mathrm{F}_{(8.443)}=.28, \mathrm{p}<.001\right)$. Se observa como a menor edad mayor índice de lesión ( $\beta=-.15$, $p=.004)$, a mayor experiencia deportiva mayor índice de lesión $(\beta=-.22$, $\mathrm{p}<.001)$. Por otra parte, existen diferencias significativas con respecto al tipo de modalidad deportiva (deporte individual o deporte colectivo) siendo los deportes colectivos los que tuvieron mayor índice de lesión (1.64/.64) ( $\beta=-.359, p<.001$ ). El género no tuvo ninguna relación significativa con el índice de lesión.

En la tabla 5 se aprecia una regresión lineal donde se relacionan los factores deportivos y personales con el número de lesiones leves.

En la tabla 5 se aprecia que las variables que más contribuyen en la varianza (15.70\%) son: edad, tipo modalidad y la experiencia. La combinación de estas variables predice de forma significativa el número de lesiones leves $\left(\mathrm{F}_{(8.443)}=11.53, \mathrm{p}<.001\right)$.

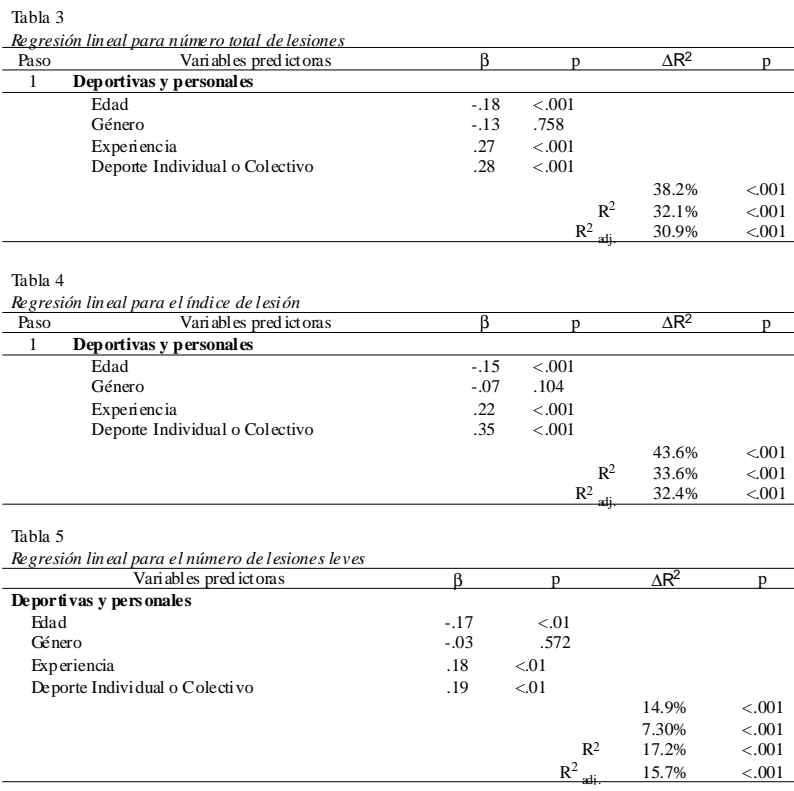

Un 14.94\% de la varianza puede ser predicha por estos factores deportivos y personales. La combinación de estas variables predice de forma significativa el número de lesiones leves $\left(\mathrm{F}_{(8.43)}=.12, \mathrm{p}<.001\right)$. Se puede observar como a menor edad mayor índice de lesión ( $\beta=-.17$, $\mathrm{p}=.002$ ). Por otro lado, se observa que a mayor experiencia deportiva mayor número de lesiones leves ( $\beta=-.18, p=.004)$. Por otra parte, existen diferencias significativas con respecto al tipo de modalidad deportiva (deporte individual o deporte colectivo) siendo los deportistas de equipo los que tuvieron mayor número de lesiones leves (2.47/1.13) ( $\beta=-.19, p=.004$ ). El género no tuvo ninguna relación significativa con el número de lesiones leves.

En la tabla 6 se indica una regresión lineal donde se relacionan los factores deportivos y personales con el número de lesiones moderadas. Las variables que más contribuyen en la varianza (35.1\%) son, edad, experiencia y tipo de modalidad. La combinación de estas variables predice de forma significativa el número de lesiones moderadas $\left(\mathrm{F}_{(8.433)}=31.30, \mathrm{p}<.001\right)$.

Un 54.6\% de la varianza puede ser predicha por estos factores deportivos y personales. La combinación de estas variables predice de forma significativa el número de lesiones moderadas $\left(\mathrm{F}_{(8.433)}=.33, \mathrm{p}<.001\right)$. En la tabla 6 se puede observar como a menor edad mayor número de lesiones moderadas ( $\beta=-.10, p=.042$ ), y a mayor experiencia deportiva mayor número de lesiones moderadas $(\beta=-.275, \mathrm{p}<.001)$. Por otra
Tabla 6

\begin{tabular}{|c|c|c|c|c|c|}
\hline Paso & Variables pred ictoras & $\beta$ & $\mathrm{p}$ & $\Delta \mathrm{R}^{2}$ & $\mathrm{p}$ \\
\hline \multirow[t]{9}{*}{1} & \multicolumn{5}{|l|}{ Dep ortivas y personales } \\
\hline & Edad & -.10 & $<.05$ & & \\
\hline & Género & -.06 & .380 & & \\
\hline & Experiencia & .27 & $<.001$ & & \\
\hline & Deporte Individual o Colectivo & .40 & $<.001$ & & \\
\hline & & & \multirow{4}{*}{$\mathrm{R}^{2}{ }^{\mathrm{R}}$} & $54.6 \%$ & $<.001$ \\
\hline & & & & $5.7 \%$ & $<.001$ \\
\hline & & & & $36.1 \%$ & $<.001$ \\
\hline & & & & $35.1 \%$ & $<.001$ \\
\hline \multicolumn{6}{|c|}{ Tabla 7} \\
\hline \multicolumn{6}{|c|}{ Regresión lineal para la suma del número de lesiones graves y muy graves } \\
\hline & Variables pred ict oras & $\beta$ & $\mathrm{p}$ & $\Delta R^{2}$ & $\mathrm{p}$ \\
\hline \multicolumn{6}{|c|}{ Deportivas y personales } \\
\hline \multicolumn{2}{|c|}{ Edad } & -.04 & .410 & & \\
\hline \multicolumn{2}{|c|}{ Género } & -.05 & .222 & & \\
\hline \multirow{5}{*}{\multicolumn{2}{|c|}{$\begin{array}{l}\text { Experiencia } \\
\text { Deporte Individual o Colectivo }\end{array}$}} & .14 & $<.05$ & & \\
\hline & & -.48 & .487 & & \\
\hline & & & & $3.6 \%$ & $<.001$ \\
\hline & & & $\mathrm{R}^{2}$ & $1.6 \%$ & $<.001$ \\
\hline & & & $R^{2}$ & $9.0 \%$ & $<.001$ \\
\hline
\end{tabular}

parte, existen diferencias significativas con respecto al tipo de modalidad deportiva siendo los deportes colectivos los que tuvieron mayor número de lesiones moderadas (2.43/.66) $(\beta=.40, \mathrm{p}<.001)$. El género no tuvo ninguna relación significativa con el número de lesiones leves.

Por último, en la tabla 7 se indica una regresión lineal donde se relacionan los factores deportivos y personales con la suma del número de lesiones graves y muy graves.

Los datos de la tabla 6 señalan que la variable que más contribuye en la varianza (9.0\%) es la experiencia. La combinación de esta variables predice de forma significativa el número de lesiones graves y muy graves $\left(\mathrm{F}_{(8.433)}=6.59, \mathrm{p}<.001\right)$.

Un $3.6 \%$ de la varianza puede ser predicha por este factor deportivo. La experiencia deportiva predice de forma significativa el número de lesiones graves y muy graves $\left(\mathrm{F}_{(8.433)}=.03, \mathrm{p}<.001\right)$. En la tabla 7 se observa como a mayor experiencia deportiva mayor número de lesiones graves y muy graves $(\beta=-.14, p<.05)$. Por otra parte, ni el género, ni la modalidad deportiva ni la edad tuvieron ninguna relación significativa con el número de lesiones graves y muy graves.

\section{Discusión}

Para conocer la vulnerabilidad del deportista a la lesión se consideró apropiado estudiar la relación entre el número de lesiones totales (frecuencia y gravedad) con las variables sociodemográficas (edad, género, experiencia y modalidad deportiva). Según los resultados de este estudio, los deportistas de deportes colectivos se lesionan más que los deportistas individuales. En la misma línea, las investigaciones revelan que la práctica de deportes colectivos, sobre todo aquellos en los que existe contacto, entraña un mayor índice de lesiones en sus practicantes (Nicholl, Coleman y Williams, 1995; Ytterstad, 1996).

No se encontraron diferencias en cuanto al género. Por el contrario, en el estudio de Bahr y Krosshaug (2005), dirigido por la National Collegiate Athletic Association (NCAA) por medio del seguimiento de lesiones (Injury Surveillance System) se obtuvieron los siguientes resultados: las jugadoras de baloncesto se lesionaron el ligamento cruzado anterior con una frecuencia 7 veces mayor que los hombres durante los entrenamientos y cinco veces mayor durante los partidos. Por lo contrario, en el estudio de Olmedilla, Andreu, Ortín y Blas (2009) los resultados muestran que las lesiones producidas durante los entrenamientos duplicaban a las producidas en partidos.

Por otra parte, Powell y Barber-Foss (1999) determinaron que las mujeres sufren con más frecuencia que los hombres ciertos tipos de lesiones de rodilla, específicamente las que afectan al ligamento cruzado anterior (LCA), en dos deportes (baloncesto y fútbol). La mayoría de estas lesiones del ligamento cruzado anterior se clasifican como lesiones sin contacto, es decir, no son producto de una colisión con un oponente o contra un objeto inanimado. Son lesiones propias de deportes en que se practican rápidos cambios de dirección o desaceleración en carrera, o repetidos saltos y aterrizajes. Algunos trabajos como el de Hardy y Riehl (1988) sugieren que las diferencias de género y la especificidad del deporte practicado podrían influir en la relación entre estrés y lesión, 
aunque, en contraste con los resultados de este estudio, las diferencias de género no han aparecido, como ocurrió en el estudio de Ford, Eklund y Gordon (2000).

En lo referente a la experiencia deportiva, en este estudio los sujetos que tenían más experiencia tuvieron mayor número de lesiones totales y mayor índice de lesión, probablemente debido a la mayor cantidad de tiempo practicando deporte, $y$ por ende, a la posibilidad de poder haber sufrido una mayor cantidad de lesiones. El estudio de la relación entre la historia de lesiones de los deportistas y su influencia en las variables psicológicas de éstos, tiene como objetivo conocer el grado de influencia de la historia lesional, y en qué aspectos lo hace en la psicología del deportista (Abenza, Olmedilla y Ortega, 2010; Olmedilla, Prieto y Blas, 2009; Short et al., 2004). Por otro lado, la historia previa de lesiones del deportista ha sido un factor ampliamente estudiado en este contexto (Armason, Sigurdsson, Gudmundsson, Holme y Bahr, 2004; Krucera, Marshall, Kirkendal, Marchak y Garret, 2005; Rodal et al., 2013).

Algunos autores señalan que entre el 20\% y el 25\% de las lesiones son recaídas de alguna lesión anterior (Hawkins y Fuller, 1999). Por otro lado, Krucera et al. (2005), en un estudio con futbolistas jóvenes, señalan que la historia previa de lesiones es un factor relevante en la probabilidad de volverse a lesionar. En este sentido indican que los futbolistas que han sufrido una lesión previa, tienen 2,6 veces más probabilidades de lesionarse que jugadores sin historia previa de lesión. En la misma línea, Rodal et al. (2013) destacan la relevancia de esta variable en relación con la probabilidad de volverse a lesionar.

Según el modelo de Andersen y Williams (1988), las lesiones previas pueden influir en posteriores lesiones. En este sentido, Grove y Prapavessis (1992), descubrieron que las lesiones previas estaban relacionadas con las lesiones presentes ocurridas en hospitales. Por otro lado, Armason et al. (2004), en un estudio con 306 futbolistas señalan la edad y las lesiones anteriores como principales factores de riesgo.

Respecto a los deportistas con menor edad, en este estudio se observó que tenían mayor número de lesiones totales y mayor índice de lesión. Por lo contrario, otros estudios que relacionan la influencia de la edad y la categoría, parecen indicar que a mayor edad existe una mayor probabilidad de lesión en el jugador (Majewski, Susanne y Klaus, 2006; Schmidt-Olsen, Jorgensen, Kalund y Sorensen, 1991). Según Bahr y Krosshaug (2005) la edad interacciona con la variable género, siendo los más propensos a la lesión los menores entre 10-19 años y los mayores entre 40-49 años (Bahr y Krosshaug, 2005).

En lo referente a la etiología de las lesiones en los deportes, Bahr y Krosshaug (2005) acentúan la necesidad de utilizar un «modelo comprensivo», considerando todos los posibles acontecimientos que conducen a la lesión (situación, comportamiento del jugador y del opositor y biomecánica del cuerpo entero). Según este modelo, basado en el trabajo epidemiológico de Meeuwisse (1994), no solo se incluye una perspectiva biomecánica, sino también las características del deporte, los factores intrínsecos y extrínsecos. En un estudio que relacionaba lesiones, género y edad de diferentes deportistas del Oeste de Australia, se indica que eran incapaces de determinar en qué medida ocurrían las lesiones (Stevenson et al., 2000). A pesar de esto, había relativamente pocas lesiones graves registradas, quizá debido a que los deportistas evaluados eran aficionados.

Sería recomendable la utilización de instrumentos específicos para el registro de lesiones, y cumplimentados por fisioterapeutas, rehabilitadores, etc.). En este sentido, las sugerencias realizadas por Junge et al. (2000) respecto a la utilización de un sistema estandarizado de registro y evaluación de las lesiones proporcionaría criterios muy válidos de comparación de resultados; así como criterios más eficaces para definir exactamente el nivel de gravedad de la lesión y el tipo de lesión desde diferentes puntos de vista (mecanismos de lesión, lesiones crónicas, o quizá, enfermedades específicas relacionadas con el deporte). Probablemente, como se hizo en el estudio de Abenza et al. (2010) si definiéramos los deportistas lesionados a todos aquellos que habían padecido una lesión grave o muy grave, y a los no lesionados como a todos aquellos que no habían sufrido lesión o bien que la lesión había sido leve o moderada (de escasa influencia en la práctica deportiva), nos encontraríamos diferencias, contradictorias o no, entre los dos grupos.

Por último, se considera que aspectos como el partido o el entrenamiento, el tiempo de juego, los tipos de entrenamiento, la posición de juego, la edad, la categoría, y la historia de lesiones anteriores, pueden ser variables que se pueden combinar en futuras investigaciones (Hawkins y Fuller, 1999).

\section{Conclusiones}

En este estudio se planteó como objetivo, determinar la relación entre el número de lesiones totales (frecuencia y gravedad) con las variables sociodemográficas (edad, género, experiencia y modalidad deportiva). En el presente estudio se aportan resultados que incrementan la fundamentación teórica sobre lesiones y factores personales y deportivos, siendo de gran utilidad para comprender mejor la relación entre estos aspectos y las lesiones deportivas. A continuación, se enumeran las principales conclusiones:

1. Un mayor número de lesiones totales y un mayor índice de lesión se asocia con una menor edad y con una mayor experiencia, probablemente debido a la mayor cantidad de tiempo practicando deporte de forma continuada, y por ende, a la posibilidad de poder haber sufrido una mayor cantidad de lesiones. Teniendo en cuenta que estos resultados solo pueden ser extrapolables para la muestra de este estudio, puede que los sujetos de edades inferiores entrenen durante más tiempo, obteniendo un mayor índice de lesión (número de lesiones totales/ horas de entrenamiento semanal) como se indica en los resultados, aumentando el riesgo de sobreentrenamiento y de lesión. Por otro lado, los sujetos con mayor experiencia son los que se sitúan en un mayor rango de edad, pudiendo ser mayor el historial de lesiones.

2. Un mayor número de lesiones totales y de índice de lesión se asocia con deportes colectivos, probablemente porque en la práctica de los deportes colectivos estudiados (baloncesto, balonmano y fútbol) existe mayor contacto físico que en los deportes individuales (tenis, natación y atletismo) entrañando una mayor frecuencia de lesión entre sus practicantes.

Anexo I: Registro de lesiones

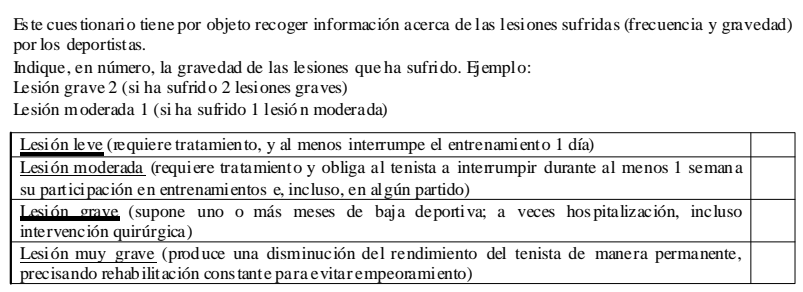

precisando rehabilitación cons tante para evitar empeorami ento)

Anexo II: Cuestionario de recogida de información de aspectos sociodemográficos y personales

Este cuestionario tiene por objeto recoger información acerca de aspectos sociodemográficos y personales. Toda la información recogida en este cuestionario es confidencial sirviendo únicamente a los fines de la investigación a la que va dirigida. Por favor, se ruega leer con deteni miento y contestar con sinceridad.

\begin{tabular}{|c|c|c|c|}
\hline \multicolumn{2}{|l|}{ Club: } & Sexo H / M & \begin{tabular}{l|l} 
& Federado SI / NO \\
\end{tabular} \\
\hline Edad: & Peso: & Talla: & ¿Cuál es tu deporte? \\
\hline Categoría: & & $\begin{array}{l}\text { lo en horas por semana: }<1 \text { hora } \\
\text { ras } / 5-8 \text { horas }>\text { de } 8 \text { horas }\end{array}$ & $\begin{array}{l}\text { Tiempo jugando a tu deporte: }<1 \text { año/1- } \\
4 \text { años/4-8 años/8-12 años/>de } 12 \text { años }\end{array}$ \\
\hline
\end{tabular}

\section{Referencias}

Abenza, L., Olmedilla, A. y Ortega, E. (2010). Efectos de las lesiones sobre las variables psicológicas en futbolistas juveniles. Revista Latinoamericana de Psicología, 42(2), 265-277.

Andersen, M.B. y Williams, J.M. (1988). A model of stress and athletic injury: Prediction and prevention. Journal of Sport and Exercise Psychology, 10, 294-306.

Armason, A., Sigurdsson, S.B., Gudmundsson, A., Holme, I. y Bahr, R. (2004). Physical fitness, injuries, and team performance in soccer. Medicine \& Science in Sports \& Exercise, 36(2), 278-85.

Bahr, R. y Krosshaug, T. (2005). Understanding injury mechanisms: a key component of preventing injuries in sport. British Journal of 
Sports Medicine. 39, 324-329.

Buceta, J.M. (1996). Psicología y lesiones deportivas: Prevención y recuperación. Madrid: Dykinson.

Buceta, J.M. (2008). Aproximación psicológica a la lesión deportiva. Documento presentado en el I Simposium Nacional de Psicología del Deporte, Lesiones deportivas, prevención y rehabilitación, Universidad Católica San Antonio de Murcia, Murcia, España.

Díaz.P.; Buceta J.M. y Bueno, A.M (2002). Estrés y vulnerabilidad a las lesiones deportivas. Selección, 11(2), 86-94

Ekstrand, J., Hägglund, M. and Waldén, M. (2009) Injury incidence and injury patterns in professional football: the UEFA injury study. British Journal of Sports Medicine, 45(7), 553-558.

Ford, I. W., Eklund, R. C., y Gordon, S. (2000). An examination of psychosocial variables moderating the relationship between life stress and injury time-loss among athletes of a high standard. Journal of Sports Sciences, 18(5), 301-312.

Grove, J.R. y Prapavessis, H. (1992). Preliminary evidence for the reliability and validity of an abbreviated Profile of Mood States. International Journal of Sport Psychology, 23, 93-109.

Hanson, S., McCullagh, P. y Tonymon, P. (1992). The relationship of personality characteristics, life stress, and coping resources to athletic injury. Journal of Sport and Exercise Psychology, 14, 262-272.

Hardy, C. J., y Riehl, R. E. (1988). An examination of the life stressinjury relationship among noncontact sport participants. Behavioral Medicine, 14, 113-118.

Hawkins, R.D. y Fuller, C.W. (1999). A prospective epidemiological study of injuries in four English professional football clubs. British Journal of Sports Medicine, 33(3), 196-203.

Heil, J. (1993). Psychology of Sport Injury. Champaign, IL: Human Kinetics.

Jan, M.D., Margareta, M., Birgitta, O. y Sten-Otto, M.D. (1983). Incidence of soccer injuries and their relation to training and team success. The American Journal of Sports Medicine, 11, 63-67.

Kucera, K.L., Marshall, S.W., Kirkendall, D.T., Marchak, P.M. y Garrett, W.E. (2005). Injury history as a risk for incident injury in youth soccer. British Journal Sport Medicine, 39, 462-466.

Majewski, M., Susanne, H. y Klaus, S. (2006). Epidemiology of athletic knee injuries: A 10-year study. Knee, 13(3), 184-188.

Martínez-Romero, J.L. (2008). Medicina deportiva y lesión: prevención y recuperación. I Simposium Nacional de Psicología del Deporte, Lesiones deportivas, prevención y rehabilitación. Murcia: Universidad Católica San Antonio de Murcia.

Meeuwisse, W.H. (1994). Assessing causation in sport injury: a multifactorial model. Clinical Journal of Sport Medicine, 4(3), 166170.

Naylor, J.L. (2008). The Role of Mental Training in Injury Prevention. Athletic Therapy Today, 14(2), 27-29.
Nicholl, J.P., Coleman, P. Y Williams, B.T. (1995). The epidemiology of sports and exercise related injury in the United Kingdom. British Journal of Sports Medicine, 29(4), 232-239.

Olmedilla,A., Ortega, E. y Abenza, L. (2007). Percepción de los futbolistas juveniles e influencia del trabajo psicológico en la relación entre variables psicológicas y lesiones. Cuadernos de Psicología del Deporte, 7(2), 75-87.

Olmedilla, A., Andreu, M.D., Ortín, F.J. y Blas, A. (2009). Lesiones y fútbol base: un análisis en dos clubes de la Región de Murcia. Retos: Nuevas tendencias en Educación Física, Deporte y Recreación, 16, 63-66.

Olmedilla, A., Prieto, J.M. y Blas, A. (2009). A history of injuries and their relationship to psychological variables in tennis players. Annuary of Clinical and Health Psychology, 5, 67-74.

Palmeira, A. (1998). Antecedentes psicológicos de la lesión deportiva. Revista de Psicología del Deporte, 8(1), 117-132.

Palmi, J. (2001). Visión psicosocial en la intervención de la lesión deportiva. Cuadernos de psicología del deporte, 1(1), 69-79.

Parlebás, P. (1988). Elementos de sociología del deporte. Unisport: Málaga.

Powell, J.W., Barber-Foss, K.D. (1999). Injury patterns in selected high school sports: A review of the 1955-97 seasons. Journal of Athletic Training, 34, 227-284.

Rodal, F., García Soidán, J.L. y Arufe, V. (2013). Factores de riesgo de lesión en atletas. Retos: Nuevas tendencias en Educación Física, Deporte y Recreación, 23, 70-74.

Schmitd-Olsen, S., Jorgensen, U., Kalund, S. y Sorensen, J. (1991). Injuries among young soccer players. American Journal of Sports Medicine, 19(3), 273-275.

Short, S.E., Reuter, J., Brandt, J., Short, M.W., y Kontos, A.P. (2004). The Relationships among three components of perceived risk of injury, previous injuries and gender in contact sport athletes. Athletic Insight, 6(3).

Spano, J.L. (2008). Impact of life stressors on athletes. Athletic Therapy Today, 13 (2), 42-43.

Stevenson, M.R., Hamer, P. y Finch, C.F. (2000). Sport, age, and sex specific incidence of sports injuries in Western Australia. British Journal Sports Medicine, 34, 188-94.

Thomas, J.R. y Nelson, J.K. (2007). Métodos de investigación en actividad física. Barcelona: Paidotribo.

Van Mechelen, W.H. y Kemper, H.C. (1992). Incidence, severity, aetiology and prevention of sports injuries. A review of concepts. Sports Medicine, 14(2), 82-99.

Ytterstad, B. (1996). The harstad injury prevention study: the epidemiology of sports injuries. British Journal of Sports medicine, 30(1), 64-68.

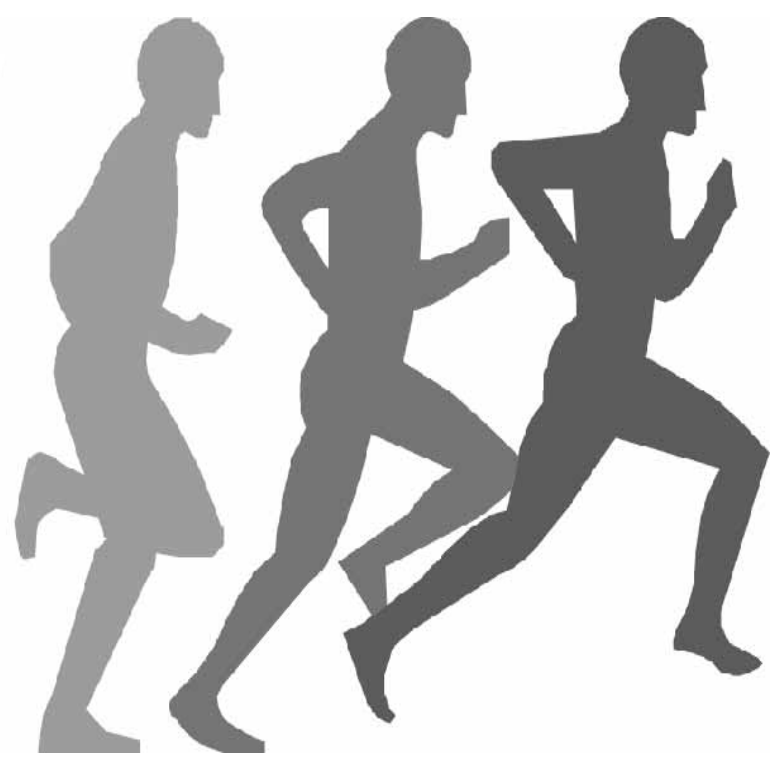

